\title{
Photographic Sky Surveys and Cataclysmic Variables: Recent progress
}

\author{
René Hudec*t \\ Czech Technical University in Prague, Faculty of Electrical Engineering, Technicka 2, CZ 160 \\ O0 Prague, Czech Republic, and Kazan Federal University, Russian Federation \\ E-mail: rene.hudec@gmail.com
}

\begin{abstract}
We report on an application of astronomical photographic plate archives in study of cataclysmic variables and related inventory and digitization of archival astronomical plates including metadata. These archives represent huge database of historical astronomical data, astrometric, photometric, and spectroscopic, with in general many applications in modern astrophysics.
\end{abstract}

The Golden Age of Cataclysmic Variables and Related Objects - III, Golden2015

7-12 September 2015

Palermo, Italy

*Speaker.

${ }^{\dagger}$ I acknowledge grant 13-33324S by GACR 


\section{Introduction}

The astronomical plate archives represent the only method how to study the behavior of the CVs (and other astrophysical objects in general) over very long (100 years or even more) time intervals, and the only method to go back in time. In addition, huge monitoring times (up to 30 $000 \mathrm{hrs}$ of continuous monitoring) are available allowing to detect and to study rare events such as outbursts. The databases allow to study prominent spectra and/or spectral changes as well (Hudec et al., 2012).

In the understanding of physical processes and evolution in cataclysmic variables, long-term coverage plays an important role, as well as dense coverage in order to monitor brightenings and outbursts. Historical archival data, mostly photographic, are here of large value. The photography was widely used to record images and spectra in astronomy for more than 100 years. As a result, large collections of astronomical photographic records exist at many worlds observatories and astronomical institutes (Hudec 1999 and 2005). My estimate based on personal visits and works with many different plate collections (more than 60) is that there are more than 8 million astronomical photographic negatives worldwide (Hudec, 1999, Hudec 2005, Hudec and Hudec, 2013). We are aware that there are still numerous photographic astronomical archives hidden to the community, so the actual number of astronomical negatives may be much higher.

The basic photographic substrates common in astronomical photographic archives are as follows. (i) Photographic glass negatives and (ii) Photographic negatives on plastic substrates. Both types are useful fo $\mathrm{CV}$ research.

The other division is according to the image/target type recorded on the negative. The basic separation is between direct images and spectral images, where the spectral images can be either single or multiple spectra, or wide field low dispersive spectra with objective prism. The direct images may be single (each star one image) or multiple (each star is recorded several times on the same plate). The negatives were in the past used with large number and rich variety of astronomical telescopes and cameras, and correspondingly, the type variety of archived negatives is also very rich. Many collections represent wide field sky images, but there are also large collections of small negatives, both glass and plastics, taken in the focal plates of medium and large telescopes.

There are numerous important astronomical plate archives in many countries, including plate collections that are little known to the community and that have been little investigated in the past. Within the framework of a Czech-US collaborative project, we have analyzed some of them, obtaining test scans with the use of a portable digitizing device. Digitization is a necessary step for an extended evaluation of the plate data using dedicated programs and powerful computers. Several recently found large US negative archives such as Baker-Nunn and/or Super Baker Schmidt are expected to play especially important role in high-energy astronomy. More recently, we investigated European, Russian (Kazan) and Chinese astronomical plate archives.

We also note that the historical astronomical photographic archives represent important part of historical and cultural heritage and, in many cases, reflects and document important epochs of history of science and technology at relevant Institute or Observatory and/or country. Many collections are related to names famous from the history of astronomy and astrophysics. 


\section{The CV astrophysics with photographic plates}

The astronomical plates represent important source of valuable data for many areas of recent astronomy and astrophysics. They include huge amount of information as single photographic plate may contains up to 100000 star images. Most of plates and negatives were never before investigated in full detail, as before computer era this was virtually impossible. The recent wide plate scanning together with dedicated software allows perfect and complex data mining in these archives for the first time.

In CVs research, the photographic data archives represent valuable data source for: (i) longterm photometric monitoring over very long time intervals (up to 100 years), (ii) dense photometric coverage to reveal outbursts and brightenings, and (iii) spectral investigations (including spectral changes and spectral time variations), both low-dispersive as well as high-dispersive. Very valuable are relatively numerous high-dispersive spectra of historical novae, located at several observatories, and dated back to about 1912 (Figs. 1, 2, 3). Obviously these spectra were never investigated with modern techniques.

In the CVs science, the optical monitoring by photographic archival plates enables to: (i) identify the type of the system, (ii) place the events (e.g. outbursts) in the context of the longterm activity of the system, and (iii) form the representative ensemble of events (e.g. outbursts and transitions between the states) in (a) a given system, and/or (b) in a type of systems.

We note that the transitions between the CVs activity states (e.g. outbursts, high/low states) are often fast and unpredictable. Archival photographic plates enable the possibility to study this activity on very long timescales (even more than 100 years), including search for possible large changes of the activity character. Large changes of activity of some X-ray binary systems (of various types) on timescale of decades can be detected and investigated, as the photographic data can significantly extend the mapped time interval.

Photographic data also enable to study the optical counterparts even in the time intervals before the discovery of these objects. Unpredictable and rare events (outbursts, transitions between the states) can be also discovered on the archival photographic plates. Digitization and use of dedicated software and computers allows effective data mining in this direction.

Transients with the large-amplitude brightening (e.g. novae) can be discovered on the photographic plates as the new objects (transients). We note that the large-amplitude activity of such objects can be studied even on the non-digitized photographic plates (e.g. by Argelander method + microscope). It is also possible to combine the photographic archival data with the more recent CCD observations, including transformations to the same band (e.g. V) are possible.

The recently investigated extended negative collections with very dense coverage and excellent time resolution (down to $2 \mathrm{sec}$ ) represent especially excellent database for $\mathrm{CV}$ research. These archives include Baker Super Schmidt and Baker Nunn negatives, as well as Hewitt camera plates/films. The Baker Super Schmidt camera domed films typically cover 55 deg diameter FOV, with lim mag 15, and provide dense sampling of $20 \mathrm{sec}$. There are $\sim 110000 \mathrm{such}$ negatives, with $\sim 10$ years time coverage. The Baker-Nunn camera networks negatives have FOV 30 x 5 deg, lim mag is 16 , and offer very dense sampling of $\sim$ few sec. The total number of these frames exceeds 1 mil negatives, with more than 20 years time coverage. 


\section{The astronomical photographic plate archives}

The astronomical archival plate collections that we visited and investigated during last 5 years include those housed in the following 16 US institutions: Carnegie Observatories Pasadena, CA, Lick Observatory, CA Yerkes Observatory, WI, Mt Palomar Observatory, CA, PARI, Rosman, NC (which has a collection of plates from many observatories), KPNO Tucson, AZ, CFHT Waimea, HI, IfA Manoa, HI, USNO Flagstaff, AZ, USNO Washington, DC, Steward Observatory Tucson, AZ, NMSU, Las Cruces, NM, Rosemary Hill Observatory, University of Florida, Gainesville, FL And Leander McCormick Observatory, University of Virginia, Charlottesville, VA, Smithsonial Archives Washington, DC, and Hazy Space Center, Dulles, VA. Our estimate is that there are more than 2 million astronomical archival plates and/or negatives in these archives. There are however many US plate collections not included in our study (Robbins and Osborn, 2009). We performed a quality check and analyzed these plate archives with emphasis on their scientific, historical and cultural value, which we have found to be enormous.

In addition to that, collaboration with several German Institutes with astronomical plate archives started, with emphasis on digitization and investigation of relevant photographic collections. In the recent project, we have visited and digitized samples of astronomical photographic databases e.g. in Torino, Vatican Castel Gandolfo, Catania, Merate, Asiago, Bologna, Loiano, OHP Haute Provence, Nice/Calern, and more recently in China, Red Purple Observatory in Nanjing, and Shanghai/Sheshan, and in 2017 the plate archive at the Tuorla Observatory, Finland, was fully digitized in just one week, with 11000 plates. The main science behind was to obtain database to study optical behavior of celestial X-ray sources (important for synergy with long-term X-ray monitoring with Lobster Eye Telescopes) but the data have general use including CV research.

In 2016, the plate archive of the Kazan Federal University at the Engelhardt Astronomical Observatory (EAO, about 20000 plates taken both in Kazan as well as in various observing stations, including more than 600 worlds unique plates of Moon with stars) was visited and inspected for quality and applications. The glass photographic plate library at EAO represents a relatively large archive of photographic plates, covering the period of more than 90 years, and obtained by different telescopes: 1) A photographic survey of the sky (FON), plate size $30 \mathrm{~cm}$ x $30 \mathrm{~cm}-1746$ plates; 2) Photographic catalog (size $30 \mathrm{~cm} \times 30 \mathrm{~cm}$ ) - 700 plates; 3) The Moon with Stars - 612 plates; 4) Positive images - 272 plates; 5) Comets, asteroids, moons - 159 plates; Schmidt Telescope: 6) Selected Areas of Kapteyn - 1500 plates; 7) Comets and asteroids - 950 plates; 8) Radio sources - 253 plates; 9) Variable Stars -350 plates; 10) New and supernovae - 300 plates; 11) Random objects - 250 plates; Meniscus telescope : 12) Selected Areas of Kapteyn - 1070 (2130) plates. Total: 10292 catalogued plates, but the total estimated number of plates in the archive is larger, about 15000 .

\section{Photographic plate scanning methods}

Before digitization era, the access to the data recorded in astronomical photographic plates and negatives was difficult. Recently, there are digitization efforts at several observatories, but the data in vast majority of them are still unavailable. The three basic plate scanning techniques developed and tested over last decade are as follows. 
- Custom made high quality scanner, mostly with granite table. Preference: very high astrometric accuracy 0.1 microns or less, drawbacks: very expensive device, expensive operation, plate scanning cost 100 Euro per plate or more.

- Commercial high quality flatbed scanner. Most used instrumentation in plate scanning. Moderately expensive investment and operation, one plate scan typically of order of 10 Euro. Usually good accuracy in one direction but worse in the direction of CCD line movement where typically waves are detectable.

- High quality digital camera with homogeneous light table and high quality lens. Moderate expensive equipment but operation is extremely fast typically one plate just very few seconds and hence very inexpensive. Typical cost of digitization is just 0.5 Euro or even less. Suitable for medium sized up to small and very small plates where the method provides very good resolution and accuracy.

\section{In situ digitization with digital camera}

Most of the plate archives that we visited have no plate scanners and lack modern instrumentation in general. As our study includes plate digitization, it was necessary to find a solution. Since we were going to travel from Europe to the US by air, the obvious option was a transportable digitization device based on a digital camera with a high-quality lens and a stable tripod. This solution has the following advantages over other techniques: the device is easily transportable, and offers much faster scanning and higher repeatability than commercial flatbed scanners, because there are no moving scanner parts. The equipment that we used at the 1st stage of the project was as follows: Camera: 21 MPx Canon EOS 5D Mark II, Lenses: Canon EF 24-70 f/2.8 L USM and Canon 70-200mm F4, a stable tripod, and a Fomei LP-310 professional photographic light table. More recently, a better custom-made light table based on highly homogeneous LED illumination was used, and also a further improved camera (39 Mpx) and lenses. The recorded images are then corrected for lens image distortions and for other effects, in order to store research-grade digital images. The achieved pixel size depends on the size of the plate, and fine pixel sizes of order of 25-30 microns or less can be typically obtained for small and medium sized plates (up to $16 \mathrm{~cm} \mathrm{x}$ $16 \mathrm{~cm}$ or $13 \mathrm{~cm}$ x $13 \mathrm{~cm}$ ) while for larger plates larger pixel sizes will be obtained (or, alternatively, the large plate may be covered by several shots to achieve smaller pixel sizes). The best results are provided for small (few $\mathrm{cm} \mathrm{x}$ few $\mathrm{cm}$ ) plates such as high dispersive spectra and direct images taken with large telescopes, then the angular pixel resolution achieved is of order of 5 microns or even better. Note that 5 microns is already near the grain size of photographic emulsion.

\section{The inventory of astronomical plate collections}

There is no complete inventory of all astronomical photographic records available. The wide field astronomical plate database WFPDB includes only collections wide field sky images (but there are numerous small narrow-field photographic plates as well taken at focal planes of large telescopes before the CCD era) end even that is incomplete. In the USA, the inventory of north 


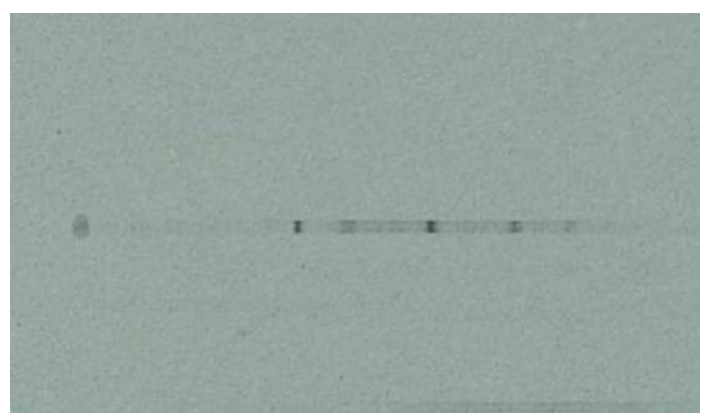

Figure 1: Example of digitized small narrow field spectral photographic plate taken in focal plane of large telescope of the Catania Observatory, image digitized by digital camera, pixel size 5 microns. Spectrum of Nova Geminorum 1912.

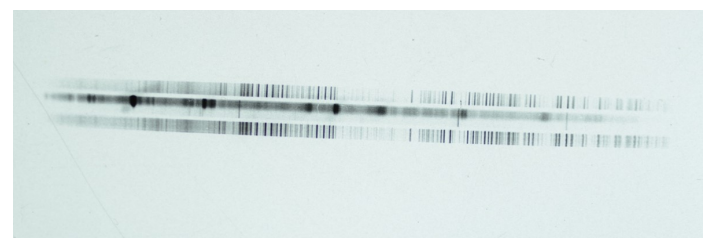

Figure 2: Example of digitized small narrow field spectral photographic plate taken in focal plane of large telescope of the Asiago Observatory, image digitized by digital camera, pixel size 5 microns. Spectrum of historical nova.

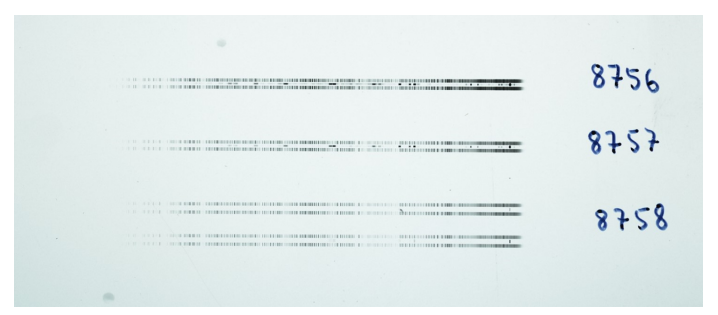

Figure 3: Example of digitized small narrow field spectral photographic plate taken in focal plane of large telescope of the Asiago Observatory, image digitized by digital camera, pixel size 5 microns. Spectrum of historical nova. Typically the spectral time evolution is covered by about 50 spactra taken at various epochs.
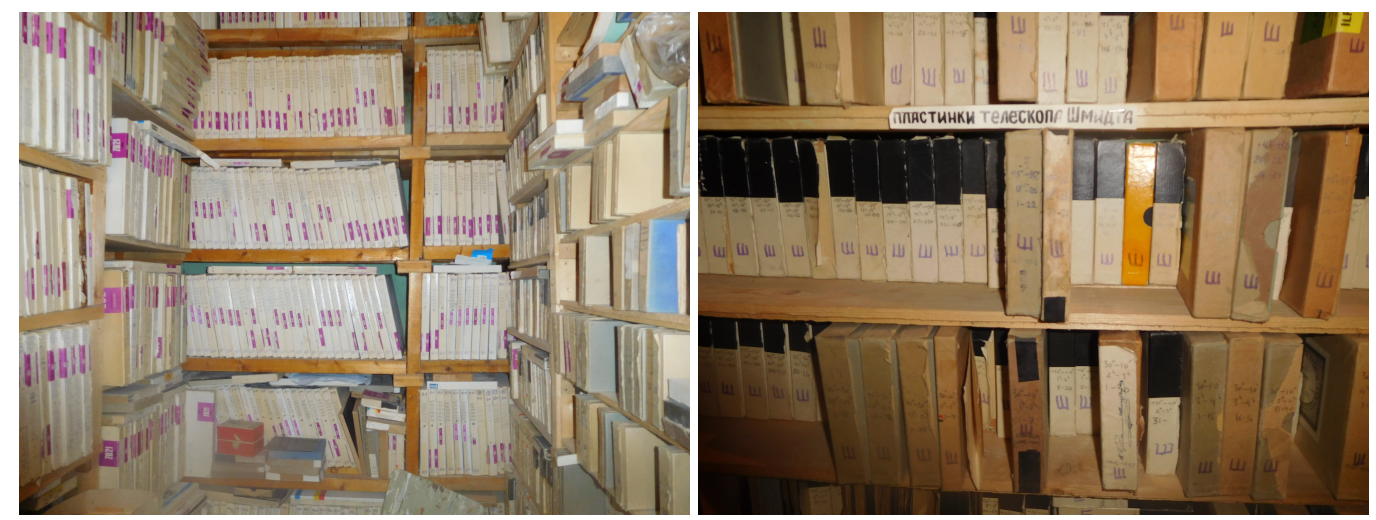

Figure 4: Recently visited astronomical plate archive at the Engelhardt Astronomical Observatory near Kazan, Russian Federation. 
American plates was compiled by Robbins and Osborn (2009), but the list is again far from complete.

Our experience shows that there are numerous astronomical plate collections, some of them large, at places and Institutions unknown to the community before (Fig. 4). Some of them were visited by us with test plate scanning using our transportable device. Few archives were digitized by us completely (e.g. Tuorla Observatory, Finland, and UNAM Mexico)

\section{Conclusions}

The scientific value of historical astronomical data recorded in photographic sky archives, including CVs astrophysics, is enormous. On the other hand, the digitization efforts are still limited to few places. It is obvious, that, taking the huge number of astronomical archival plates and negatives as well as limited resource into account, we need inexpensive and fast in situ digitization technique.

The total number of plates is higher than expected-in many of the locations, the actual number of plates is unknown. As no catalogs exist, the real number of plates is very difficult to estimate, but for sure the places that we visited only in the USA have more than 2 million photographic plates and/or negatives in their collections. We plan to continue these efforts, now with emphasis on German and European astronomical plate collections. In addition to that we have started collaboration with Chinese and Russian archives as well.

We plan to continue data mining and plate digitization in astronomical photographic plate/negative archives is as follows. (i) Digitize the plate archives using a fast and transportable scanning device, as described above. This scanning method is fast and inexpensive. These are important considerations, as the archives are scattered and there are very large numbers of plates. (ii) Create electronic catalogs. (iii) Include these catalogs into search programs like WFPD, operated by our Bulgarian colleagues (e.g. Tsvetkov et al., 2005, and Tsvetkov, 2009).

For CV research, the major importance of these data sets is (i) large time coverage for photometry, (ii) dense coverage (for observing outbursts and rare light changes), and (iii) spectroscopy and spectral changes/evolution.

\section{Acknowledgements}

We acknowledge GA CR grant 13-33324S.

\section{References}

[1] Hudec, R. et al., Acta Polytechnica, IBWS2011 Proceedings, 1(52), 2012.

[2] Hudec, R. Astrophysics with Astronomical Plate Archives, in Exploring the Cosmic Frontier: Astrophysical Instruments for the 21st Century. ESO Astrophysics Symposia, European Southern Observatory series. Edited by Andrei P. Lobanov, J. Anton Zensus, Catherine Cesarsky and Phillip J. Diamond. Series editor: Bruno Leibundgut, ESO. ISBN 978-3-540-39755-7. Published by Springer-Verlag, Berlin and Heidelberg, Germany, 2007, p.79

[3] Hudec, R., An introduction to the world's large plate archives, Acta Historica Astronomiae, vol. 6, p. 28-40, 1999. 
[4] Robbins, L.; Osborn, W., The Census of Astronomical Photographic Plates in North America, Preserving Astronomy's Photographic Legacy: Current State and the Future of North American Astronomical Plates. ASP Conference Series, Vol. 410. Edited by Wayne Osborn and Lee Robbins. San Francisco: Astronomical Society of the Pacific, 2009., p.81

[5] Hudec, R., World's Astronomical Plate Archives: Past, Present, Future in Virtual Observatory: Plate Content Digitization, Archive Mining and Image Sequence Processing, iAstro workshop, Sofia, Bulgaria, 2005 proceedings, ISBN-10 954-580-190-5, p. 197-208

[6] Hudec R. and Hudec L., Finding Hidden Treasures: Investigations in US Astronomical Plate Archives Acta Polytechnica, Vol. 53, No. 3, p.23, 2013 [4] 